\title{
EXPRESSION OF KI-67 (MIB-1) AND GLUT-1 PROTEINS IN NON-ADVANCED PROSTATIC CANCER
}

\author{
Elzbieta Luczynska ${ }^{1}$, AnNa Gasinska², Waclaw WilK ${ }^{3}$
}

${ }^{1}$ Department of Radiology, Centre of Oncology, Maria Sklodowska-Curie Memorial Institute Cracow, Poland 2Department of Applied Radiobiology, Center of Oncology, Maria Sklodowska-Curie Memorial Institute Cracow, Poland

${ }_{3}^{3}$ Department of Tumour Pathology, Centre of Oncology, Maria Sklodowska-Curie Memorial Institute Cracow, Poland

\begin{abstract}
The expression of Ki-67 (MIB-1) and glucose transporter-1 (GLUT-1) were evaluated in patients with clinically localized prostate cancer (PC) who had undergone radical prostatectomy with curative intent.

140 low advanced PC specimens were studied. Protein expression was assessed immunohistochemically on tumour sections and expressed as a labelling index, i.e. the percentage of positively stained cells. In the case of $\mathrm{Ki}-67$ nuclear staining and in the case of GLUT-1 membrane and cytoplasmic staining was considered as positive.

The patients' mean age was $62.9 \pm 6.2$ years. There were $13(9.3 \%)$ at pTNM stage $1,78(55.7 \%)$ at stage $2,40(28.6 \%)$ at stage 3 and $9(6.4 \%)$ at stage 4 , respectively. $75(53.6 \%)$ tumours were well differentiated (Gleason score $\leq 6), 52(37.1 \%)$ moderately differentiated (Gleason score of 7) and $13(9.3 \%)$ poorly differentiated (Gleason score 8-10). The mean pre-operative serum PSA was $9.9 \pm \mathrm{SE} 0.5 \mathrm{ng} / \mathrm{ml}$, and the mean LI was equal to $8.1 \pm 0.6 \%$ and $29.7 \pm 2.0 \%$, for MIB- 1 and GLUT-1, respectively. Increase of pathological tumor volume and tumor grade was associated with statistically significant growth of PSA ( $p<0.011$ ) and ${ }_{\text {MIB-1 }}$ LI ( $p<0.003$ ), however, for GLUT-1 LI the relation was not significant. Ki-67 expression was correlated with PSA levels $(\mathrm{p}=0.013)$ and GLUT-1 scores $(\mathrm{p}=0.04)$.

In $\mathrm{PC}$, an increase in the proliferation rate (higher ${ }_{\mathrm{MIB}-1} \mathrm{LI}$ ) in higher $\mathrm{pTNM}$ stages and tumour grades may point to $\mathrm{Ki}-67$ as a good marker of biological aggressiveness useful in selecting patients for more aggressive treatment. A correlation between proliferation and GLUT-1 score may be the evidence of active glycolytic metabolism in hypoxic regions.
\end{abstract}

Key words: GLUT-1, immunohistochemistry, Ki-67, MIB-1, prostatic cancer.

\section{Introduction}

Early identification of curable PC may result in improved cure rates and potentially increased life expectancies. The vast majority of cancers are diagnosed within the PSA range of 3.0-9.9 $\mathrm{ng} / \mathrm{ml}$, with biopsy Gleason score 6 or 7 , and clinical tumour stage T1-T2, but the biological behaviour of these tumours varies considerably. Some might have been treated unnecessar- ily as the post-operative prognostic features proved highly favourable, while others might present a disease too advanced to cure. Pathological and clinical methods are not sufficient to identify patients for proper treatment. Therefore, PC, which is already metastatic at diagnosis or able to produce metastasis in effect of its histological grade, must be distinguished from indolent prostate cancer tumours [1]. Patients with aggressive tumours should be candidates for adjuvant therapy, be- 
fore or after surgery and radiotherapy, and clinical decisions should be based on reliable tools capable of assessing the exact stage and aggressiveness of the disease before surgery. For this reason, there is an urgent need to develop reliable molecular methods and new prognostic markers capable of predicting disease spread at diagnosis and disease recurrence after curative intent surgery. The combination of clinicopathologic parameters and biomarkers may be very useful. The development of novel biomarkers for early cancer detection in blood or urine has the potential to improve patients' diagnosis, although many of them (e.g. alphamethylacyl-CoA racemase, or TMPRSS2-ERG6 fusion gene) are at an early stage of development and require evaluation in prospective trials [2]. Therefore, the identification of membrane antigens or biomarkers using immunohistochemical techniques has become important for the diagnosis, prognosis, classification and treatment evaluation. It was shown that it is possible to identify predictive markers for biochemical failure [3-5], disease recurrence and patient survival after radical prostatectomy $[4,6,7]$. However, there remains conflicting evidence regarding prognostic influence of an individual marker. Today, there is no unfailing indicator that can be used to foretell the disease prognosis in daily uro-oncological clinical practice.

$\mathrm{Ki}-67$ is a protein involved in cell cycle regulation and cell proliferation. The protein is expressed in proliferating cells during all active phases of the cell cycle (apart from G0). Ki-67 is confirmed to be the most promising biomarker for routine practice applications [8], which may reflect the biologically malignant potential of $\mathrm{CP}$ and justify its use in selecting candidates for active surveillance [9]. However, its significance remains controversial. Some studies showed that the proliferation fraction of primary PC, as assessed by Ki-67 (MIB-1) expression was associated with the grade or stage of prostatic cancer and clinical outcome of patients after radical prostatectomy $[1,5,10]$. However, other authors $[7,11]$ could not see prognostic impact of Ki-67 proliferative activity in their patients' set.

Glucose transporter-1 (GLUT-1), a target gene of hypoxia-inducible factor-1 (HIF-1), has been considered as a putative endogenous marker of tumour hypoxia [12]. This hypoxia-regulated membrane protein belongs to a family glucose transporters which allow the energy independent transport of glucose across the cell membrane down its concentration gradient [12] and is the most widely expressed GLUT isoform in human cancers. Elevated GLUT-1 expression has been described in many tumours including cervical carcinoma [13], and its significant influence on patients' survival was shown [14].

Some authors have studied GLUT-1 expression in $\mathrm{CP}$ cell lines $[15,16]$ and in vivo $[13,17]$, but its usefulness has not been established yet. Till now, the assessment of cell proliferation and tumour hypoxia has not yet become a standard procedure for prostate cancer management. Therefore, the aim of the study was to assess two proteins which may be a helpful adjunct for better pre-therapeutic assessment of PC aggressiveness and so contribute to improved initial patient management.

\section{Material and methods}

\section{Patients}

The study involved evaluation of 140 consecutive radical prostatectomy specimens, obtained from patients who had radical prostatectomy for localized PC between 2007 and 2011. The mean age of the patients was 62.9 \pm 6.2 years (range 49.0-77.0). Clinical staging was performed according to the American Joint Committee on Cancer (AJCC) and the International Union Against Cancer [18]. Grading was established according to the Gleason score (GS) differentiation system (range 2-10) [19]. Patients were divided into three Gleason score groups according to the American Joint Committee on Cancer (AJCC): well differentiated (Gleason score $\leq 6$ ), moderately differentiated tumours (Gleason score of 7 ) and poorly differentiated tumours (Gleason score of $8-10)$. The protocol was approved by the Ethical Committee of the Centre of Oncology, and each patient submitted written consent.

\section{Immunohistochemical assessment of tumour markers}

Following rehydration and blocking the endogenous peroxidase, in $5 \mu \mathrm{m}$ sections heat-based antigen retrieval was carried out $\left(20-60 \mathrm{~min}\right.$ at $98^{\circ} \mathrm{C}$ with $10 \mathrm{mM}$ citric acid buffer, $\mathrm{pH}$ 6.0). After 20 minutes, the sections were washed, flooded with $10 \%$ normal goat serum for $20 \mathrm{~min}$ and incubated with proper antibody overnight at $4{ }^{\circ} \mathrm{C}$. For Glut- 1 it was a rabbit monoclonal antibody (Millipore, $1: 300$ ), for $\mathrm{Ki}-67$ a mouse antiKi-67 monoclonal antibody (clone MIB-1, DAKO, $1: 75$ ) in tris-buffered saline (TBS, $\mathrm{pH}$ 7.4). After washing, slides were incubated at $37^{\circ} \mathrm{C}$ for $1 \mathrm{~h}$ with DAKO En Vision visualisation system containing goat anti-mouse (Ki-67) or goat anti-rabbit (Glut-1) IgG. The sections were stained with diaminobenzidine (DAB), counterstained with hematoxylin, dehydrated and mounted. Negative control slides omitting the primary antibody were included in each staining run. For Glut-1, positive reaction in erythrocytes within tumour capillaries provided internal positive control, however for MIB-1 it was tumour staining with known positivity. Staining intensity was evaluated by light microscopy at $\times 400$ magnification. Protein expression was shown in the form of labelling indices (LI) interpreted as a percentage of positively immunostained cells (brown) in several (5-7) malignant areas of the tissue section. Any nuclear staining regardless of intensity was 
considered positive for MIB-1. Glut-1 staining appeared in two forms, either cytoplasmic or membraneous. High-grade prostatic intraepithelial neoplasia foci and nonhyperplastic benign acini were not evaluated. Expression of GLUT-1 was assigned a score of $0-3$ : $0-$ negative $(0-<1 \%$ of tumour cells); 1 - weak (1$10 \%$ tumour cells), 2 - moderate (11-50\% of tumour cells), $3-$ strong ( $>50 \%$ of tumour cells).

\section{Statistical analysis}

Statistical analysis was performed with STATISTICA vs.9. Intergroup differences in the original data were tested with the ANOVA test or t-Student's test. P-values of less than 0.05 were considered to indicate statistical significance.

\section{Results}

We examined human histological specimens from 140 PC patients treated with radical surgery. There were $13(9.3 \%)$ at pTNM stage $1,78(55.7 \%)$ at stage 2 , $40(28.6 \%)$ at stage 3 and $9(6.4 \%)$ at stage 4 , respectively. In the analysed group there were 75 (53.6\%) well-differentiated, $52(37.1 \%)$ moderately differentiated and $13(9.3 \%)$ poorly differentiated tumours. Pre-operative PSA revealed a median PSA of $9.9 \pm 0.5$ $\mathrm{ng} / \mathrm{ml}$. PSA serum concentration significantly increased with the pTNM stage $(\mathrm{p}=0.011)$, Gleason score $(\mathrm{p}=$ $0.002)$, and tumour grade $(\mathrm{p}=0.003)$. In the three grade groups, the mean ${ }_{\text {MIB-1 }}$ LI was equal to $8.1 \pm 0.6 \%$ (Fig. 1 A). Tumour proliferation significantly increased with tumour pathological volume $(\mathrm{p}=0.002)$, Gleason score $(p=0.001)$ and grade $(p=0.0003$, Fig. $2 \mathrm{~A})$. The mean ${ }_{\text {MIB-1 }}$ LI percentages for low to high-grade groups were $6.8 \%, 8.3 \%$ and $15.4 \%$, respectively.

In $17(12.1 \%)$ out of 140 tumours GLUT-1 expression was not observed (GLUT-1 score $=0)$. The staining was weak (GLUT-1 score $=1$ ) in $24(17.1 \%)$, moderate (GLUT-1 score $=2$ ) in $66(47.1 \%)$ and strong (GLUT-1 score $=3$ ) in $33(23.6 \%)$ cases (Fig. 1B). The number of positively staining GLUT-1 cells was not correlated with pathological tumour stage $(\mathrm{p}=0.110)$, Gleason scores $(\mathrm{p}=0.346)$, and tumour grade $(\mathrm{p}=0.153)$. However, a tendency towards

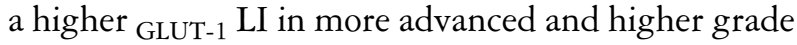
tumours (Fig. 2B) was noted. GLUT-1 expression exhibited a characteristic pattern, with staining intensity growing as a function of distance from the vascularized tumour stroma, being particularly strong in viable cell layers immediately adjacent to necrotic areas. Ki-67 expression was correlated with the PSA level $(\mathrm{p}=0.013)$ and GLUT-1 score $(\mathrm{p}=0.04)($ Fig. 3).

\section{Discussion}

The immunohistochemical expressions of $\mathrm{Ki}-67$ (MIB-1) and GLUT-1 were assessed in patients with clinically localized prostate cancer who had undergone radical prostatectomy with curative intent. The expression of the first protein was correlated with the tumour grade and volume and PSA levels, however, the second was not. We showed that cell proliferation based on $\mathrm{Ki}-67$ can distinguish a subset of aggressive tumours what reflects biologically malignant potential of $\mathrm{CP}$ and is in agreement with other authors' findings [9, 10, 20$]$. In our study ${ }_{\mathrm{MIB}-1} \mathrm{LI}$ value ranges from below 0.7 to $44.3 \%$, and was within the range obtained by other authors $[6,8,10]$, although mean value was higher than obtained in some earlier studies [9, 11]. High grade PC had significantly higher mean ${ }_{\text {MIB-1 }}$ LI than that low grade tumours, what is consistent with previous reports [7, 8, 20, 21]. However, other studies did not find any correlation between Ki-67 (MIB-1) expression and higher score and/or advanced pathological stage [1].
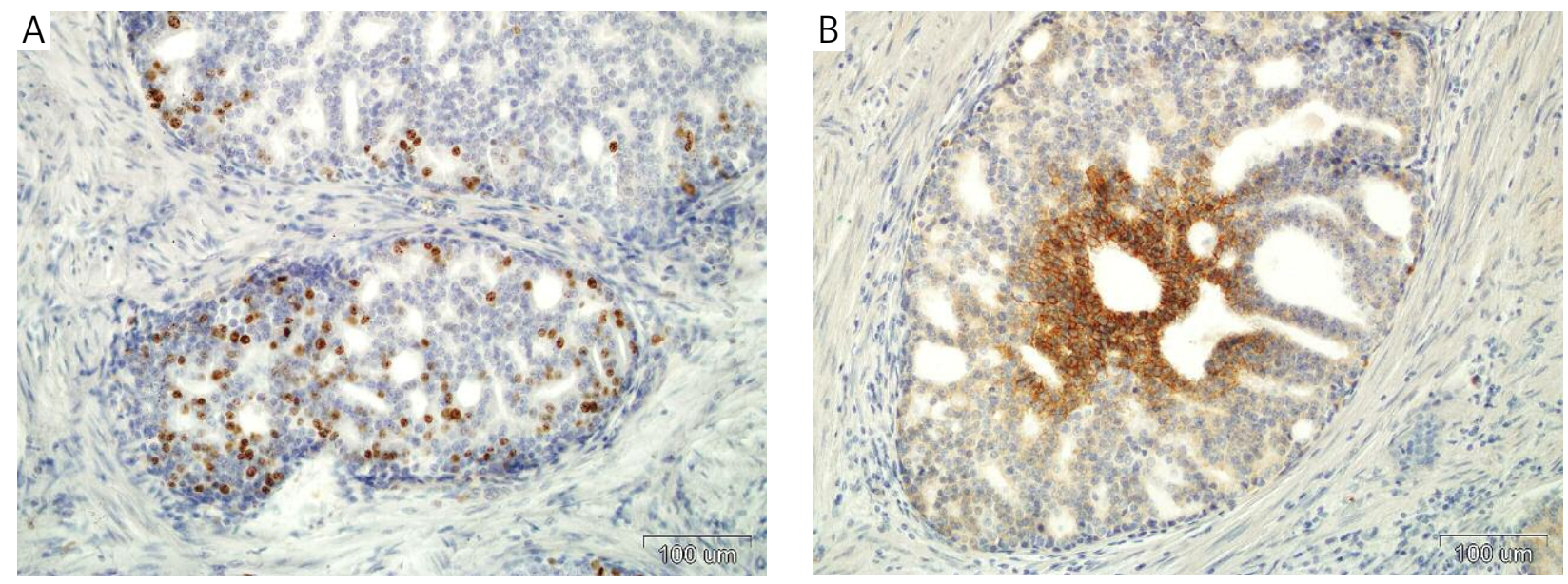

Fig. 1. Strong expression of Ki-67 (MIB-1) (A) and GLUT-1 (B) in a moderately grade prostate carcinoma. Glut-1 expression exhibited a characteristic pattern, with staining intensity increasing as a function of distance from the vascularised tumour stroma 

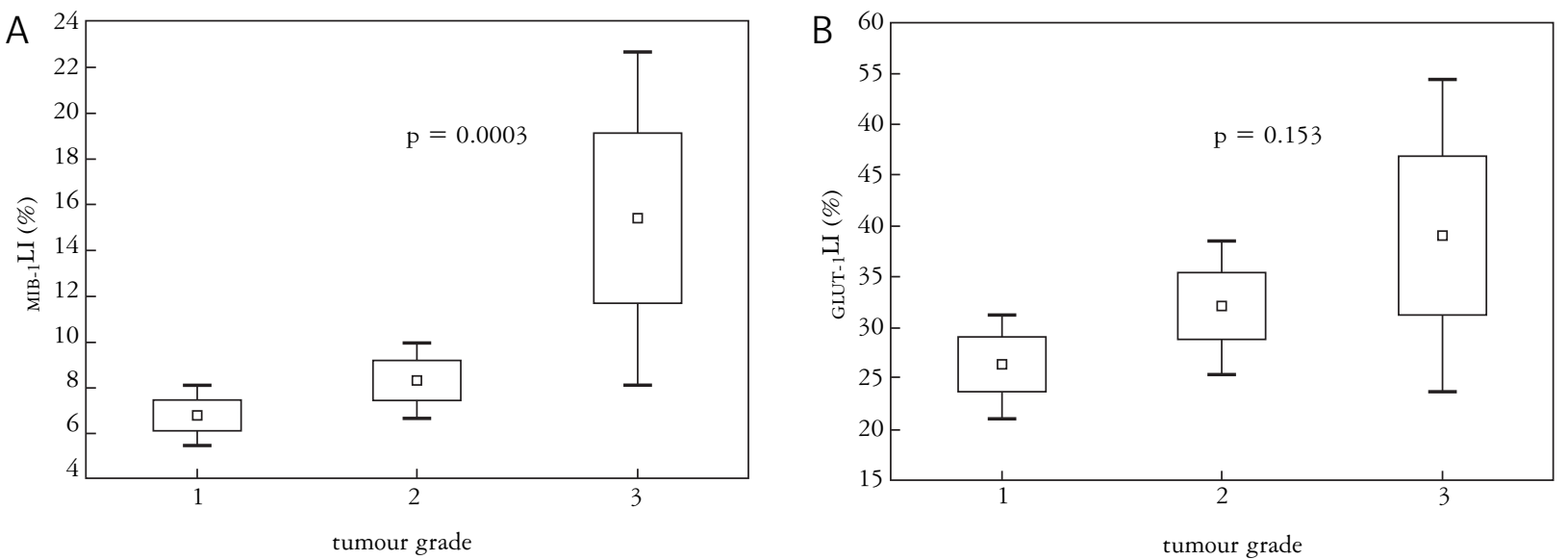

Fig. 2. Association between MIB-1 LI (A) and GLUT-1 LI(B) and PC tumour grade. Symbols represent mean values \pm SE

Indicating a higher MIB-1 expression in high grade tumours may allow to distinguish aggressive from indolent tumours. Furthermore, it is suggested that Ki-67 may be less prone to misinterpretation related to heterogeneity or interpretational difficulties than Gleason score in the case of small amounts of cancer in the biopsies [10].

In our study, the Ki-67 expression was positively correlated with the PSA level which is a parameter commonly used in clinical practice for indicating tumour advancement. Recently, it has been shown that a high $\mathrm{Ki}-67$ expression level was associated with clinical progression and a tendency towards higher rates of PSA relapse in multivariate analysis [21]. It is known that androgen plays a central role in prostate cancer development and proliferation [22]. Testosterone required to drive PSA production may also induce PC cell proliferation.

In our study, MIB-1 expression was also correlated with GLUT-1 scores. Overexpression of glucose transporters, specially Glut-1, is a common event in human malignancies. It is known that the major adaptive cell response to hypoxia is the metabolic switch from oxidative to glycolytic metabolism. Increased glucose consumption is a basic characteristic of malignant cells and is linked to higher energy production from aerobic glycolysis [23]. This metabolic way holds a high level of lactate production, even in the presence of oxygen. The major function of aerobic glycolysis is to maintain high levels of glycolytic intermediates to support anabolic reactions (biosynthesis of proteins, lipids, nucleic acids) in cells. This explains why increased glucose metabolism is selected in proliferating cells [24].

In our set of tumours, big heterogeneity in GLUT-1 expression and a broad range of results (0-89\%) was observed. It is difficult to compare our findings with other authors because expression of GLUTs in prostate tumour specimens has been characterized poorly, and not many quantitative data are available. Reinicke et al. [25] indicated a very low level of GLUT-1 expression in 28 clinical specimens of PC. Chandler et al.

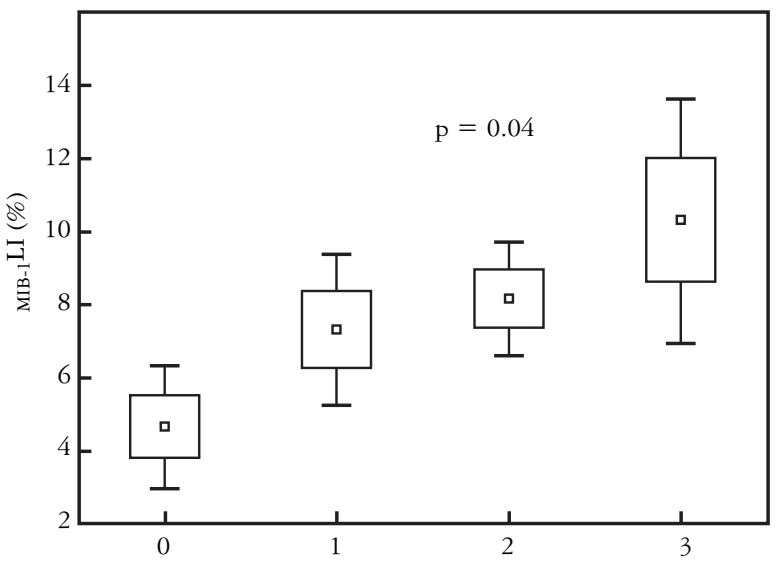

Fig. 3. Correlation between prostate tumour cell proliferation (MIB-1 LI) and hypoxia (GLUT-1 score). Symbols represent mean values $\pm \mathrm{SE}$

[16] showed only one positive GLUT-1 staining out of three clinical specimens analysed. In Airley et al. [13] study on tissue microarrays, expression of GLUT-1 in malignant prostate tissue was absent. A question is whether any array design or microdissected material for gene analysis includes representative tissue samples for analysis. This is especially important in the case of prostate cancer because it may contain many small areas of malignancy. More data are available on PC cell lines $[15,16]$. In this study, no correlation was found between GLUT-1 expression and grade of differentiation. The expression level increased with the advancing grade of malignancy, but did not reach statistical significance (probably due to a low number of grade 3 tumours). However, other authors [17] observed a positive correlation between GLUT-1 expression and grade but they analysed it at the mRNA level and no attempt was made to analyse the expression of GLUT-1 protein. Methodological inconsistencies, however, could 
be explained by the fact that the presence of mRNA does not always correlate with the expression of the corresponding protein.

In our set of tumours, GLUT-1 expression was not correlated with the tumour size, what is known that tumour oxygenation is independent of tumour size. We observed an association between GLUT-1 expression and proliferation - ${ }_{\text {MIB-1 }} \mathrm{LI}$, which increased with intensity of GLUT staining (GLUT-1 score). In the case of intraductal CP, because of lower blood supply as the cells proliferate to the gland centre and get far from the vasculature surrounding the glandular compartment, over-expression and preferential localization of GLUT-1 to specific cellular adaptations of the plasma membrane might be a potential mechanism to assure more efficient nutrient supply to the cancer epithelial cells during their intra-glandular growth. This may show that proliferation and hypoxia are not exclusive and proliferating cells may be found within areas of hypoxic staining in some of the tumours [26]. Our findings support a role for Glut-1 in PC proliferation and need of elevated energy requirements of prostate carcinoma cells [16], what was shown on PC cell lines [27].

Overexpression of GLUT-1 may find application as a marker of malignancy in PC as it is correlated with grade $[15,16]$ and also with proliferation, what was shown in our study and reflects the adaptive upregulation of anaerobic glycolysis that may ultimately promote tumour cell survival [24]. However, further studies will be required to determine the relationship between mRNA and protein levels of glucose transporters [16].

\section{Conclusions}

In PC, an increase of proliferation rate (higher ${ }_{\mathrm{MIB}-1} \mathrm{LI}$ expression) in higher pTNM stages and tumour grades may point to $\mathrm{Ki}-67$ as a good marker of biological aggressiveness suitable for patients' selection for more aggressive treatment. A correlation between proliferation and GLUT-1 score may be the evidence of active glycolytic metabolism in hypoxic regions.

This work was supported by the Polish Ministry of Science and Higher Education (grant No N403 240837). The authors declare no conflict of interest.

\section{References}

1. Revelos K, Petraki C, Gregorakis A, et al. p27 kip1 and Ki-67 (MIB) immunohistochemical expression in radical prostatectomy specimens of patients with clinically localized prostate cancer. In Vivo 2005; 19: 911-920.

2. Killick E, Bancroft E, Kote-Jarai Z, Eeles R. Beyond Prostatespecific Antigen - future biomarkers for the early detection and management of prostate cancer. Clin Oncol 2012: 24: 545-555.

3. Goto T, Nguyen BP, Nakano M, et al. Utility of Bcl-2, P53, $\mathrm{Ki}-67$, and Caveolin-1 immunostaining in the prediction of biochemical failure after radical prostatectomy in a Japanese population. Urology 2008; 72: 167-171.
4. Cowen D, Troncoso P, Khoo VS, et al. Ki-67 is an independent correlate of biochemical failure in prostate cancer treated with radiotherapy. Clin Cancer Res 2002; 8: 1148-1154.

5. Malhotra S, Lapointe J, Salari K, et al. A tri-marker proliferation index predicts biochemical recurrence after surgery for prostate cancer. Plos ONE 2011; 6: 1-7.

6. Inoue T, Segawa T, Shiraishi T, et al. Androgen receptor, Ki67, and $\mathrm{p} 53$ expression in radical prostatectomy specimens predict treatment failure in Japanese population. Urology 2005; 66: 332337.

7. Vis AN, Noordzij MA, Fitoz K, et al. Prognostic value of cell cycle proteins $\mathrm{p} 27$ (kip) and MIB-1, and the cell adhesion protein CD44s in surgically treated patients with prostate cancer. J Urol 2000; 164: 2156-2161.

8. Berney DM, Gopalan A, Kudahetti S, et al. Ki-67 and outcome in clinically localized prostate cancer: analysis of conservatively treated prostate cancer patients from Trans-Atlantic Prostate Group study. Brit J Cancer 2009; 1000: 888-893.

9. Nagao K, Yamamoto Y, Hara T, et al. Ki67 and BUBR1 may discriminate clinically insignificant prostate cancer in the PSA range $<4 \mathrm{ng} / \mathrm{ml}$. Jpn J Clin Oncol 2011; 41: 555-564.

10. Zellweger T, Gunther S, Zlobec I, et al. Tumour growth fraction measured by immunohistochemical staining of Ki67 is an independent prognostic factor in preoperative prostate biopsies with small-volume or low-grade prostate cancer. Int J Cancer 2009; 124: 2116-2123.

11. Stapleton AM, Zbell P, Kattan MW, et al. Assessment of the biologic markers p $53, \mathrm{Ki}-67$, and apoptotic index as predictive indicators of prostate carcinoma recurrence after surgery. Cancer 1998; 82: 168-175.

12. Macheda ML, Rogers S, Best JD. Molecular and cellular regulation of glucose transporter (GLUT) proteins in cancer. J Cell Physiol 2005; 202: 654-662.

13. Airley R, Evans A, Mobasheri A, Hewitt SM. Glucose transporter Glut-1 is detectable in peri-necrotic regions in many human tumor types but not normal tissues: Study using tissue microarrays. Ann Anatomy 2010; 192: 133-138.

14. Airley R, Loncaster J, Davidson S, et al. Glucose transporter Glut1 expression correlates with tumor hypoxia and predicts metastasis-free survival in advanced carcinoma of the cervix. Clin Cancer Res 2001; 7: 928-934.

15. Effert P, Beniers AJ, Tamimi Y, et al. Expression of Glucose Transporter 1 (Glut-1) in cell lines and clinical specimens from human prostate adenocarcinoma. Anticancer Res 2004; 24: 3057 3064.

16. Chandler JD, Williams ED, Slavin JL, et al. Expression and localization of GLUT-1 and GLUT12 in prostate carcinoma. Cancer 2003; 97: 2035-2042.

17. Stewart GD, Gray K, Pennington CJ, et al. Analysis of hypoxiaassociated gene expression in prostate cancer: lysyl oxidase and glucose transporter-1 expression correlate with Gleason score. Oncology Rep 2008; 20: 1561-1567.

18. AJCC Cancer Staging Manual. Edge SB, Byrd DR, Compton CC, et al. (eds). Springer, New York - Dordrecht - Heidelberg - London 2010; 457-464.

19. Gleason DF. The Veterans Administration Cooperative Urological Research Group: histological grading and clinical staging of prostatic carcinoma. In: Urologic Pathology: The prostate. Tannenbaum M (ed.). Lea and Febiger, Philadelphia 1997; 171-197.

20. Lopez-Beltran A, Cheng L, Blanca A, Montironi R. Cell proliferation and apoptosis in prostate needle biopsies with adenocarcinoma Gleason score 6 or 7. Anal Quant Cytol Histol 2012; 34: 61-65. 
21. Liu Y, Vlatkovic L, Saeter T, et al. Is the clinical malignant phenotype of prostate cancer a result of highly proliferative immuneevasive B7-H3-expressing cell population? Int J Urol 2012; 19: 749-756.

22. Wang Y, Kreisberg JI, Ghosh PM. Cross-talk between the androgen receptor and the phosphatidylinositol 3-kinase/Akt pathway in prostate cancer. Curr Cancer Drug Targets 2007; $7: 591$ 604.

23. Warburg O. On the origin of cancer cells. Science 1956; 123: 309-314.

24. Lunt SY, Vander Heiden MG. Aerobic glycolysis: meeting the metabolic requirements of cell proliferation. Annu Rev Cell Dev Biol 2011; 27: 441-464.

25. Reinicke K, Sotomayor P, Cisterna P, et al. Cellular distribution of Glut-1 and Glut- 5 in benign and malignant human prostate tissue. J Cell Bioch 2012; 113: 553-562.

26. Hoskin PJ, Sibtain A, Daley FM, et al. The immunohistochemical assessment of hypoxia, vasculaity and proliferation in bladder carcinoma. Radiother Oncol 2004; 72: 159-168.

27. Singh G, Lakkis CL, Laucirica R, Epner DE. Regulation of prostate cancer cell division by glucose. J Cell Physiol 1000; 180: 431-438.

\section{Address for correspondence}

\section{Anna Gasinska}

Department of Applied Radiobiology

Center of Oncology, Maria Sklodowska-Curie

Memorial Institute

Garncarska 11

31-115 Cracow, Poland

tel. +48124231073

fax +48124226680

e-mail: z5gasins@cyf-kr.edu.pl 